\title{
Strategies to improve continuous positive airway pressure compliance: A review
}

\author{
Christina Michele DiNapoli \\ Weill Cornell Medical College Center for Sleep Medicine, New York, USA.
}

Correspondence: Christina Michele DiNapoli. Address: Weill Cornell Medical College Center for Sleep Medicine, New York, USA. Email: chd9077@med.cornell.edu

Received: January 7, 2014

DOI : $10.5430 /$ jnep.v4n7p62
Accepted: March 4, $2014 \quad$ Online Published: April 24, 2014

URL: http://dx.doi.org/10.5430/jnep.v4n7p62

\section{Abstract}

Continuous positive airway pressure (CPAP) is the gold standard of treatment for patients diagnosed with significant Obstructive Sleep Apnea (OSA). Although CPAP is highly effective in preventing the repetitive upper airway collapses in OSA, compliance to treatment is poor, which in turn leads to poorer health outcomes. In this study an extensive review of the literature was completed using the Cochrane library, CINAHL, PubMed and Embase databases. The research objective of this study was to determine the efficacy of interventions designed to increase compliance with CPAP. The themes that were found to be effective in increasing compliance with CPAP were increased patient education and intensive patient support. Improvements in CPAP compliance will positive impact patients, patient's families and the health care system. In conclusion, the evidence points to the necessity of implementation of an intensive patient education and patient support program for all OSA patients.

\section{Key words}

Obstructive Sleep Apnea (OSA), Continuous positive airway pressure (CPAP), Compliance, Adherence, Adult, Education, Sleep Apnea

\section{I ntroduction}

Obstructive sleep apnea (OSA) is a common sleep disorder that requires lifetime care. OSA is characterized by repetitive obstructive airway closures during sleep, which are associated with fragmented sleep and oxygen desaturations ${ }^{[1]}$. OSA has been shown to negatively impact health. Untreated OSA is associated with an increased risk of cardiovascular and cerebrovascuar diseases ${ }^{[3]}$. Excessive daytime sleepiness, a consequence of the sleep fragmentation from airway closures, has been shown to adversely impact quality of life. Moreover, OSA has been shown to be associated with impaired cognition, memory, mood alterations as well as decreased occupational and functional capacity ${ }^{[4]}$.

Continuous Positive Airway Pressure (CPAP) is a highly effective treatment for OSA. CPAP delivers constant positive pressure to the airway through a mask, splinting the airway open throughout the night, eliminating breathing disruptions ${ }^{[2]}$. CPAP is currently the first line treatment for patients with moderate to severe sleep apnea ${ }^{[1]}$. Despite its effectiveness, compliance with the treatment plan is poor ${ }^{[2]}$. Medicare defines CPAP compliance as $70 \%$ usage over four hours per night in any given thirty-day period ${ }^{[5]}$. Education and follow up are extremely important for all patients with chronic diseases; however, it is paramount for patients with OSA, as it can be challenging to adapt to using CPAP, limiting compliance. 
CPAP patients face various obstacles that may lead to noncompliance, ranging from lack of adequate understanding about the diagnosis to physical discomfort from CPAP.

\section{Significance of the problem}

It is estimated that $24 \%$ of the United States population has a diagnosis of OSA, however only about $4 \%$ carry the diagnosis ${ }^{[3]}$. The OSA population is growing exponentially each year, a consequence of increased public awareness and provider education about OSA ${ }^{[3,6]}$. Although CPAP is an extremely effective treatment for OSA, compliance is a critical problem and is widely recognized as a significant limiting factor in successful treatment ${ }^{[4]}$. Research shows that $30 \%-50 \%$ of patients diagnosed with OSA reject CPAP immediately and approximately $80 \%$ of OSA patients are noncompliant within a year after starting CPAP therapy ${ }^{[7]}$. Noncompliance with CPAP significantly reduces the overall effectiveness of treatment of OSA, leaving these patients at an increased risk for comorbid conditions, impaired daily functioning and decreased quality of life ${ }^{[2]}$.

OSA has a significant impact on patients and their families as well as society as a whole if not treated properly. Untreated, it leaves patients with a three-fold increase in risk for hypertension, diabetes mellitus and stroke ${ }^{[6]}$. Patients are also at an increased risk for ischemic heart disease, cardiac arrhythmia, congestive heart failure, peripheral vascular disease and valvular cardiac disease ${ }^{[8]}$. Patients with untreated OSA often experience excessive daytime sleepiness, impaired cognition and memory, decreased functional and occupational capacity as well as mood alterations, which significantly decreases quality of life ${ }^{[3,6]}$. Besides affecting the patients quality of life and health outcomes, it has been shown that untreated OSA patients have up to two times greater health care costs then similar individuals without OSA ${ }^{[9]}$.

In addition to negatively affecting the patient, untreated OSA often causes family members to suffer as well. Untreated it has been shown to have a significant effect on the patient's bed partner. As previously reported ${ }^{[3,6]}$ untreated OSA has a negative effect on the patient's bed partner's health status, sleep quality, daytime alertness, mood, overall quality of life as well as his/her personal relationship with the patient. Patients with untreated OSA are three to seven times more likely to have motor vehicle accidents and injuries than patients without the diagnosis, costing up to 16 billion dollars per year ${ }^{[10]}$. It has been estimated ${ }^{[6]}$ that patients with untreated OSA are two to three times more likely to have an occupational injury or accident, which further contributes to decreased occupational productivity and increased health costs.

Untreated sleep apnea also affects the health care system as a whole. As previously reported ${ }^{[9]}$ studies have calculated that the health related cost burden of untreated sleep apnea in the United States is approximately 3.4 billion dollars per year. These astronomical costs are typically a result of more frequent practitioner visits, increased hospitalizations, and development of comorbid conditions ${ }^{[9]}$.

Improvement of CPAP compliance will positively impact patients, patient's families, communities and the health care system as a whole. By improving CPAP compliance, patients will decrease their risk of comorbid conditions associated with untreated OSA ${ }^{[6]}$. Patient's quality of life, cognitive function as well as occupational function will also improve, as daytime symptoms improve when effectively treated by CPAP ${ }^{[3,6]}$. Moreover, research has shown that the patient's bed partner's quality of sleep, quality of life, health status, as well as relationship with the patient improves when a patient is compliant with CPAP ${ }^{[3,6]}$. With increased CPAP compliance, patients will experience less daytime sleepiness, reducing their increased risk of accidents and poor job performance, which in turn has benefits for the community. It has been estimated that 567,000 collisions and 980 fatalities annually could be avoided by effective CPAP treatment ${ }^{[10]}$. Importantly, research has shown that effectively treating OSA will decrease health care system costs as it reduces follow up care required for comorbitities associated with untreated OSA ${ }^{[9]}$. It becomes extremely important to evaluate what interventions are most effective in increasing CPAP compliance among adult patients diagnosed with OSA. 


\section{Search strategy}

The research objective of this search was to determine the efficacy of interventions designed to increase compliance with CPAP. Many mechanical, psychological and educational interventions have been proposed to increase CPAP compliance in patients diagnosed with OSA. Searches were carried out in the Cochrane Library, CINAHL, PubMed and Embase databases. The PICO (population, intervention, comparison, and outcome) format was used to choose the search terms as it helps to focus on certain key concepts ${ }^{[11]}$. The PICO format helps to narrow down the key concepts of the question, and choose search terms based off of the key concepts ${ }^{[11]}$. The search terms that were used were "Obstructive Sleep Apnea", “Continuous Positive Airway Pressure”, "Noncompliance” and "Nonadherence”.

After combining the four main searches together a total of 8,618 articles were produced. The search was then restricted to the English language and duplicates were eliminated, which produced 6,269 articles. After restricting the articles to the English language, a title review on the remaining articles was carried out. The inclusion criteria consisted of articles that focused on the evaluation of interventions to improve CPAP compliance in adult patients who were diagnosed with OSA. Exclusion criteria included articles that discuss AutoCPAP, Bi-Level Positive Airway Pressure (BiPAP), Auto Servo Ventilation (ASV), Mandibular Advancement Devices, weight loss as well as ENT surgical procedures as primary treatments for OSA. Articles that focused on patients under the age of 18 were excluded, as the patient population being assessed was adults. After performing the title review of the articles to evaluate if they met inclusion or exclusion criteria 191 articles were kept. The abstract of each article was read and reviewed to see if it met all inclusion criteria, and at the end of the abstract review 76 articles were kept. Each of the 76 articles were read in full to further review if each individual article met the inclusion criteria and if it helped to assess interventions to improve CPAP compliance. After the article review, 28 articles were left for evaluation (see Figure 1).

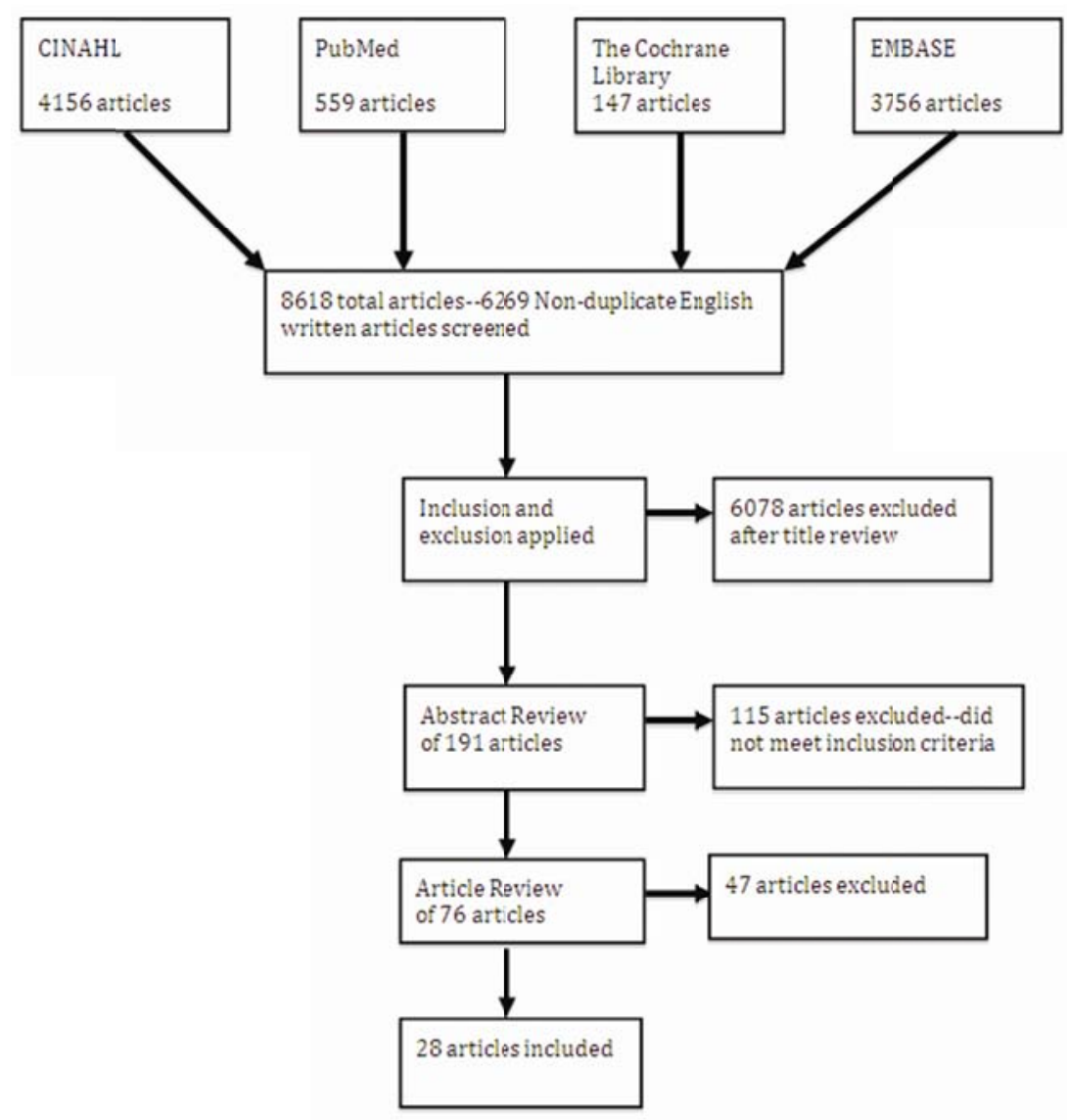

Figure 1. PRISMA diagram 
Table 1. Johns Hopkins Nursing Evidenced-based Practice Individual Evidence Summary Tool

EBP Question: What is the most effective intervention to increase CPAP compliance among adult OSA patients? Date: $12 / 10 / 12$

\begin{tabular}{|c|c|c|c|c|c|c|}
\hline $\begin{array}{l}\text { Reference } \\
\#\end{array}$ & $\begin{array}{l}\text { Author } \\
\text { \& Date }\end{array}$ & $\begin{array}{l}\text { Evidence } \\
\text { Type }\end{array}$ & $\begin{array}{l}\text { Sample, Sample } \\
\text { Size, \& Setting }\end{array}$ & Study findings that help answer the EBP question & Limitations & $\begin{array}{l}\text { Evidence } \\
\text { Level \& } \\
\text { Quality } \\
\end{array}$ \\
\hline 37 & $\begin{array}{l}\text { Smith, I. } \\
\text { (2009) }\end{array}$ & $\begin{array}{l}\text { Systematic } \\
\text { Review }\end{array}$ & 24 RCT studies & $\begin{array}{l}\text {-Auto CPAP did not increase compliance when compared to CPAP } \\
\text {-No significant difference between BiPAP and CPAP compliance } \\
\text {-No significance in compliance with the addition of humidification } \\
\text {-Intensive support (including home visits) increase CPAP compliance } \\
\text {-CBT and education increased CPAP compliance }\end{array}$ & $\begin{array}{l}\text {-Not all studies had the } \\
\text { same methods for } \\
\text { recording compliance } \\
\text {-Not all studies had the } \\
\text { same diagnostic } \\
\text { criteria for OSA }\end{array}$ & $\begin{array}{l}\text { Level I } \\
\text { Grade A }\end{array}$ \\
\hline 23 & $\begin{array}{l}\text { Damjano } \\
\text { vic, D. } \\
\text { (2009) }\end{array}$ & RCT & $\begin{array}{l}100 \text { newly } \\
\text { diagnosed OSA } \\
\text { patients—setting-G } \\
\text { ermany }\end{array}$ & $\begin{array}{l}\text {-There was no difference in compliance between the CPAP group and } \\
\text { the Auto CPAP group } \\
\text {-Intensive support (Visit at 1, 2, } 4 \text { \& } 5 \text { months-mask fitting, } \\
\text { education and counseling given at each visit)increased CPAP } \\
\text { compliance compared to the standard support group (visit at } 3 \text { \& } 9 \\
\text { months) }\end{array}$ & $\begin{array}{l}\text {-Study was only } 9 \\
\text { months long }\end{array}$ & $\begin{array}{l}\text { Level I } \\
\text { Grade-A }\end{array}$ \\
\hline 22 & $\begin{array}{l}\text { Chervin, } \\
\text { R. } \\
\text { (1997) }\end{array}$ & RCT & $\begin{array}{l}-33 \text { subjects that } \\
\text { were new CPAP } \\
\text { users-- } \\
\text { Setting-Sleep } \\
\text { Disorders Clinic in } \\
\text { Michigan. }\end{array}$ & $\begin{array}{l}\text {-The written group (educational pamphlets on symptoms, treatment } \\
\text { and diagnosis of OSA as well as on CPAP and remedies for CPAP } \\
\text { problems) had significant increase in compliance } \\
\text {-Telephone encounter group did not show significant improvement in } \\
\text { compliance, potentially due to small sample size }\end{array}$ & $\begin{array}{l}\text {-Unequal sample size } \\
\text { between groups } \\
\text {-Follow up was } 2 \\
\text { months }\end{array}$ & $\begin{array}{l}\text { Level I } \\
\text { Grade A }\end{array}$ \\
\hline 30 & $\begin{array}{l}\text { Massie, } \\
\text { C. } \\
\text { (1999) }\end{array}$ & RCT & $\begin{array}{l}\text {-38 newly } \\
\text { diagnosed OSA } \\
\text { patients-- } \\
\text { Setting-2 suburban } \\
\text { community-based } \\
\text { sleep centers }\end{array}$ & $\begin{array}{l}\text {-Small increase in compliance was noted in heated humidification } \\
\text { patients as opposed to no humidity use } \\
\text {-No difference in compliance between patients who used heated or } \\
\text { passive humidity } \\
\text {-Patient had increased satisfaction with humidification and less side } \\
\text { effects from CPAP }\end{array}$ & $\begin{array}{l}\text {-Small sample size } \\
\text {-8 week follow up } \\
\text { period }\end{array}$ & $\begin{array}{l}\text { Level I } \\
\text { Grade A }\end{array}$ \\
\hline 28 & $\begin{array}{l}\text { Hoy, C. } \\
\text { (1999) }\end{array}$ & RCT & $\begin{array}{l}\text {-80 OSA patients- } \\
\text { Setting-Sleep } \\
\text { Center in } \\
\text { Edinburgh, U.K. }\end{array}$ & $\begin{array}{l}\text {-Intensive support and education increased CPAP compliance } \\
\text { (diagnosis explained by MD, } 20 \text { min educational video, mask fitting, } \\
\text { Call on day } 2 \text { and } 21 \text {, office visit at } 1,3 \text { and } 6 \text { months, home visit at } \\
\text { day } 7,14 \text {, and } 28 \text { and } 4 \text { months, and additional education given at } \\
\text { home } \\
\text {-Self referred group had higher compliance then partner referred } \\
\text { group did }\end{array}$ & -6 month trial & $\begin{array}{l}\text { Level I } \\
\text { Grade A }\end{array}$ \\
\hline 40 & $\begin{array}{l}\text { Sparrow, } \\
\text { D. } \\
(2010)\end{array}$ & RCT & $\begin{array}{l}-250 \text { newly } \\
\text { diagnosed OSA } \\
\text { patients- } \\
\text { Setting-2 Boston } \\
\text { Medical Centers }\end{array}$ & $\begin{array}{l}\text {-TLC-CPAP group has } 30 \% \text { higher CPAP compliance at } 12 \text { months } \\
\text { (telephone encounters were built around motivational interviewing to } \\
\text { give feedback and provide counseling, as well as address barriers and } \\
\text { poor self-efficacy-Patients called weekly for a month and monthly } \\
\text { for the next year }\end{array}$ & $\begin{array}{l}\text {-Patients enrolling in } \\
\text { study may have been } \\
\text { more inclined to } \\
\text { adhere to treatment }\end{array}$ & $\begin{array}{l}\text { Level I } \\
\text { Grade A }\end{array}$ \\
\hline 39 & $\begin{array}{l}\text { Smith, C. } \\
\text { (2006) }\end{array}$ & RCT & $\begin{array}{l}-19 \text { previously } \\
\text { noncompliant } \\
\text { CPAP patients- } \\
\text { Setting-n/a }\end{array}$ & $\begin{array}{l}\text {-The Telehealth group ( } 3 \text { sessions in week } 1 \text {, and } 1 \text { session weekly for } \\
11 \text { weeks over the phone to assess mask fit, describe how to manage } \\
\text { CPAP barriers, reinforce the consequences of noncompliance and } \\
\text { emphasize benefits of CPAP) had increased CPAP compliance then } \\
\text { the control group (discussed benefits of vitamin replacement at same } \\
\text { intervals as intervention group) }\end{array}$ & $\begin{array}{l}\text {-Small sample size } \\
\text {-Short follow up } \\
\text { period of } 12 \text { weeks }\end{array}$ & $\begin{array}{l}\text { Level I } \\
\text { Grade A }\end{array}$ \\
\hline 18 & $\begin{array}{l}\text { Ballard, } \\
\text { R. } \\
(2007)\end{array}$ & RCT & $\begin{array}{l}204 \text { previously } \\
\text { noncompliant } \\
\text { CPAP patients—n/a }\end{array}$ & $\begin{array}{l}\text {-Phase } 1-24 \% \text { of patients were compliant after the first phase of } \\
\text { treatment (mask change, addition of humidification, and saline rinses } \\
\text { were moderately helpful with compliance) - (education improved } \\
\text { compliance the most) } \\
\text {-Phase } 2 \text {-after } 90 \text { days } 28 \% \text { of patients who were previously } \\
\text { noncompliant with CPAP become compliance after switch to Bi-level }\end{array}$ & $\begin{array}{l}\text {-As these patients had } \\
\text { used CPAP in the past } \\
\text { it is possible they may } \\
\text { have been able to } \\
\text { perceive a change in } \\
\text { pressure waveform }\end{array}$ & $\begin{array}{l}\text { Level I } \\
\text { Grade A }\end{array}$ \\
\hline 24 & $\begin{array}{l}\text { DeMolles } \\
\text {, D. } \\
(2004)\end{array}$ & RCT & $\begin{array}{l}30 \text { patients newly } \\
\text { diagnosed with } \\
\text { OSA- } \\
\text { Setting-Home Care } \\
\text { Company in } \\
\text { Massachusetts }\end{array}$ & $\begin{array}{l}\text {-TLC increased CPAP usage and decreased overall sleepiness in } \\
\text { patients (modeled on Hoy et al personal intensive intervention-TLC } \\
\text { calls and monitors self-reported behavior, gives education and } \\
\text { behavioral reinforcement for target health related behaviors) }\end{array}$ & $\begin{array}{l}\text {-Small Sample size } \\
\text {-Patients had overall } \\
\text { less severe apnea } \\
\text {-Patients started out } \\
\text { with low ESS scores }\end{array}$ & $\begin{array}{l}\text { Level I } \\
\text { Grade B }\end{array}$ \\
\hline 33 & $\begin{array}{l}\text { Richards, } \\
\text { D. } \\
(2007)\end{array}$ & RCT & $\begin{array}{l}100 \text { patients } \\
\text { diagnosed with } \\
\text { OSA- } \\
\text { Setting-Sleep } \\
\text { Center in Australia }\end{array}$ & $\begin{array}{l}\text {-CBT group (2-1 hour sessions } 1 \text { week apart-PowerPoint on normal } \\
\text { sleep, daytime/nighttime consequences of OSA and effectiveness of } \\
\text { CPAP, patients encouraged to handle machine and mask during visit, } \\
15 \text { minute video with people discussing their experience with CPAP, } \\
\text { booklet with general OSA and CPAP information) showed increased } \\
\text { acceptance, usage and adherence to CPAP }\end{array}$ & $\begin{array}{l}\text {-Participants from a } \\
\text { high socioeconomic } \\
\text { area in Australia } \\
\text {-Poor statistical tests } \\
\text { chosen to evaluate data }\end{array}$ & $\begin{array}{l}\text { Level I } \\
\text { Grade B }\end{array}$ \\
\hline 25 & $\begin{array}{l}\text { Fox, N. } \\
\text { (2012) }\end{array}$ & RCT & $\begin{array}{l}75 \text { patients } \\
\text { diagnosed with } \\
\text { OSA- } \\
\text { Setting-Sleep } \\
\text { Disorders Program } \\
\text { in British Columbia, } \\
\text { Canada }\end{array}$ & $\begin{array}{l}\text {-Telemedicine group (standard care + web modem that sent } \\
\text { compliance information to a database daily-coordinator reviewed data } \\
\text { on each patient daily and if compliance was low specific intervention } \\
\text { was put into action immediately, patients had a } 4-6 \text { week follow up } \\
\text { and a } 3 \text { months follow up) had increased CPAP compliance at } 3 \\
\text { months then standard care group }\end{array}$ & $\begin{array}{l}\text {-Small sample size } \\
\text {-Only patients with an } \\
\text { ESS }>11 \text { were } \\
\text { included } \\
\text {-Only moderate to } \\
\text { severe OSA cases were } \\
\text { included }\end{array}$ & $\begin{array}{l}\text { Level I } \\
\text { Grade B }\end{array}$ \\
\hline 37 & $\begin{array}{l}\text { Smith, C. } \\
\text { (2009) }\end{array}$ & RCT & $\begin{array}{l}-97 \text { patients with } \\
\text { moderate to severe } \\
\text { OSA- } \\
\text { Setting-University } \\
\text { of Kansas Sleep Lab }\end{array}$ & $\begin{array}{l}\text {-Increased CPAP compliance in the Intervention group (music based } \\
\text { audio tape with repeated step by step instructions which guide } \\
\text { arrangement of CPAP equipment, nightly bedtime CPAP procedure } \\
\text { and relax them to sleep-in addition to an educational handout on OSA } \\
\text { and CPAP) at } 1 \text { month } \\
\text {-No difference in the } 3 \text { or } 6 \text { month follow ups }\end{array}$ & $\begin{array}{l}\text {-High attrition rate in } \\
\text { the intervention and in } \\
\text { the standard group } \\
\text {-Poor choice of } \\
\text { statistical evaluation }\end{array}$ & $\begin{array}{l}\text { Level I } \\
\text { Grade B }\end{array}$ \\
\hline 42 & $\begin{array}{l}\text { Trupp, R. } \\
\text { (2011) }\end{array}$ & RCT & $\begin{array}{l}-70 \text { patients with } \\
\text { OSA and } \\
\text { cardiovascular } \\
\text { disease- } \\
\text { Setting-Ohio Sleep } \\
\text { Center }\end{array}$ & $\begin{array}{l}\text {-Negative framed outcomes (highlighted consequences if CPAP is not } \\
\text { worn) led to increased CPAP compliance then positive framed } \\
\text { outcomes (benefits of CPAP) }\end{array}$ & $\begin{array}{l}\text {-Only patients with } \\
\text { OSA and } \\
\text { cardiovascular disease } \\
\text { were included } \\
\text {-High attrition rate }\end{array}$ & $\begin{array}{l}\text { Level I } \\
\text { Grade B }\end{array}$ \\
\hline
\end{tabular}

(Table 1 continued on page 66.) 
Table 1. (Continued.)

\begin{tabular}{|c|c|c|c|c|c|c|}
\hline $\begin{array}{l}\text { Reference } \\
\#\end{array}$ & $\begin{array}{l}\text { Author } \\
\text { \& Date }\end{array}$ & $\begin{array}{l}\text { Evidence } \\
\text { Type }\end{array}$ & $\begin{array}{l}\text { Sample, Sample } \\
\text { Size, \& Setting }\end{array}$ & Study findings that help answer the EBP question & Limitations & $\begin{array}{l}\text { Evidence } \\
\text { Level \& } \\
\text { Quality } \\
\end{array}$ \\
\hline 34 & $\begin{array}{l}\text { Roecklein } \\
\text {, K. } \\
(2010)\end{array}$ & RCT & $\begin{array}{l}-30 \text { patients } \\
\text { diagnosed with } \\
\text { OSA- } \\
\text { Setting-n/a }\end{array}$ & $\begin{array}{l}\text {-No significant difference was noted between groups } \\
\text {-If adequate size potential for personal feedback to improve CPAP } \\
\text { compliance in low socioeconomic class African Americans }\end{array}$ & $\begin{array}{l}\text {-Small sample size } \\
\text {-Results not } \\
\text { generalizable } \\
\text {-No concrete outcomes }\end{array}$ & $\begin{array}{l}\text { Level I } \\
\text { Grade C }\end{array}$ \\
\hline 41 & $\begin{array}{l}\text { Stepnows } \\
\text { ky, C. } \\
(2007)\end{array}$ & RCT & $\begin{array}{l}-45 \text { newly } \\
\text { diagnosed OSA } \\
\text { patients- } \\
\text { Setting-VA Health } \\
\text { Care System in San } \\
\text { Diego }\end{array}$ & $\begin{array}{l}\text {-Tele-monitored care (look at compliance and efficacy data, data was } \\
\text { summarized and color coded patients based on compliance } \\
\text { data-action was taken if needed) increases compliance in CPAP }\end{array}$ & $\begin{array}{l}\text {-CPAP therapists not } \\
\text { blinded to treatment } \\
\text { groups }\end{array}$ & $\begin{array}{l}\text { Level I } \\
\text { Grade C }\end{array}$ \\
\hline 44 & $\begin{array}{l}\text { Wiese, H. } \\
(2005)\end{array}$ & RCT & $\begin{array}{l}-93 \text { patients } \\
\text { diagnosed with } \\
\text { OSA- } \\
\text { Setting-University } \\
\text { of Kentucky Sleep } \\
\text { Center }\end{array}$ & $\begin{array}{l}\text {-Video group ( } 15 \text { min video education and specific information on } \\
\text { OSA) increased compliance-Video group had increased CPAP } \\
\text { compliance }\end{array}$ & $\begin{array}{l}\text {-High attrition rate in } \\
\text { both groups } \\
\text {-Poor statistical testing } \\
\text { chosen }\end{array}$ & $\begin{array}{l}\text { Level I } \\
\text { Grade C }\end{array}$ \\
\hline 27 & $\begin{array}{l}\text { Golay, A. } \\
\text { (2006) }\end{array}$ & $\begin{array}{l}\text { Quasi-exp } \\
\text { erimental }\end{array}$ & $\begin{array}{l}\text {-35 patients } \\
\text { diagnosed with } \\
\text { OSA- } \\
\text {-Setting Pulmonary } \\
\text { service at a hospital } \\
\text { in Geneva }\end{array}$ & $\begin{array}{l}\text {-Improvement in compliance with educational session (patients were } \\
\text { admitted for } 36 \text { hours for education which included sessions on } \\
\text { understanding OSA, how to use CPAP, benefits of CPAP, life with } \\
\text { CPAP, obstacles for CPAP and a round table with spouses) }\end{array}$ & $\begin{array}{l}\text {-Small sample size } \\
\text {-Not generalizable for } \\
\text { all patient populations }\end{array}$ & $\begin{array}{l}\text { Level II } \\
\text { Grade B }\end{array}$ \\
\hline 26 & $\begin{array}{l}\text { Fuchs, F. } \\
\text { (2010) }\end{array}$ & $\begin{array}{l}\text { Quasi-exp } \\
\text { erimental }\end{array}$ & $\begin{array}{l}-475 \text { patients } \\
\text { diagnosed with } \\
\text { OSA and using } \\
\text { CPAP- } \\
\text { Setting-Sleep center } \\
\text { in Germany }\end{array}$ & $\begin{array}{l}\text {-Education program group ( } 120 \text { min lecture emphasizing effects of } \\
\text { OSA and benefits of CPAP, ways to decrease CPAP side effects, self } \\
\text { support meetings and booklet summarizing information given during } \\
\text { session) increased CPAP compliance }\end{array}$ & $\begin{array}{l}\text {-High attrition rate } \\
\text {-CPAP compliance } \\
\text { was self-report }\end{array}$ & $\begin{array}{l}\text { Level II } \\
\text { Grade B }\end{array}$ \\
\hline 21 & $\begin{array}{l}\text { Cartwrigh } \\
\text { t, R. } \\
\text { (2008) }\end{array}$ & $\begin{array}{l}\text { Quasi-Exp } \\
\text { erimental }\end{array}$ & $\begin{array}{l}-10 \text { men diagnosed } \\
\text { with OSA who were } \\
\text { married- } \\
\text { Setting-Rush } \\
\text { University }\end{array}$ & $\begin{array}{l}\text {-Compliance with CPAP in married men is related to the frequency } \\
\text { with which his partner sleeps with him during his initial home } \\
\text { treatment }\end{array}$ & $\begin{array}{l}\text {-Small sample size } \\
\text {-Only married men } \\
\text { included in study } \\
\text {-Not generalizable }\end{array}$ & $\begin{array}{l}\text { Level II } \\
\text { Grade C }\end{array}$ \\
\hline 29 & $\begin{array}{l}\text { Likar, L. } \\
\text { (1997) }\end{array}$ & $\begin{array}{l}\text { Retrospect } \\
\text { ive Chart } \\
\text { Review }\end{array}$ & $\begin{array}{l}-73 \text { patients } \\
\text { diagnosed with } \\
\text { OSA- } \\
\text { Setting outpatient } \\
\text { clinic at a Veterans } \\
\text { Affairs Medical } \\
\text { Center }\end{array}$ & $\begin{array}{l}\text {-Group educational sessions ( } 2 \text { hour CPAP clinic to review data and } \\
\text { review CPAP equipment, instructed by NP about OSA and CPAP and } \\
\text { viewed an educational video on CPAP) improved CPAP compliance }\end{array}$ & $\begin{array}{l}\text {-Lack of true control } \\
\text { group due to nature of } \\
\text { design of study }\end{array}$ & $\begin{array}{l}\text { Level III } \\
\text { Grade A }\end{array}$ \\
\hline 31 & $\begin{array}{l}\text { Pamidi, S. } \\
\text { (2012) }\end{array}$ & $\begin{array}{l}\text { Retrospect } \\
\text { ive Chart } \\
\text { Review }\end{array}$ & $\begin{array}{l}-403 \text { patients with } \\
\text { OSA- } \\
\text { Setting-Chicago } \\
\text { Sleep Disorders } \\
\text { Center }\end{array}$ & $\begin{array}{l}\text {-Patients who met with a sleep specialist (discuss OSA, describe PSG } \\
\text { process, discuss pathophysiology of OSA and consequences of } \\
\text { untreated OSA, discussed importance of compliance) prior to having a } \\
\text { sleep study had increased CPAP compliance than those who met with } \\
\text { PCP }\end{array}$ & $\begin{array}{l}\text {-What was discussed } \\
\text { during PCP visit was } \\
\text { not documented }\end{array}$ & $\begin{array}{l}\text { Level III } \\
\text { Grade A }\end{array}$ \\
\hline 36 & $\begin{array}{l}\text { Silva, R. } \\
\text { (2007) }\end{array}$ & $\begin{array}{l}\text { Retrospect } \\
\text { ive Chart } \\
\text { Review }\end{array}$ & $\begin{array}{l}-1481 \text { patients } \\
\text { referred to have a } \\
\text { CPAP titration- } \\
\text { Setting Sleep } \\
\text { Institute/AFIP }\end{array}$ & $\begin{array}{l}\text {-Orientation session with a sleep technician (describing what OSA is, } \\
\text { discussed consequences of untreated OSA, how to treat OSA and a } \\
\text { mask desensitization session) prior to CPAP titration night led to } \\
\text { increase CPAP acceptance }\end{array}$ & $\begin{array}{l}\text {-Retrospective study } \\
\text { not a RCT }\end{array}$ & $\begin{array}{l}\text { Level III } \\
\text { Grade A }\end{array}$ \\
\hline 19 & $\begin{array}{l}\text { Baron, K. } \\
\text { (2012) }\end{array}$ & $\begin{array}{l}\text { Observatio } \\
\text { nal Study }\end{array}$ & $\begin{array}{l}\text { - } 31 \text { men diagnosed } \\
\text { with OSA who were } \\
\text { living with their } \\
\text { wives for over } 1 \\
\text { year- } \\
\text { Setting-University } \\
\text { of Utah }\end{array}$ & -Collaboration with wife led to increased compliance with CPAP & $\begin{array}{l}\text {-Small sample size } \\
\text {-Sample only included } \\
\text { married men }\end{array}$ & $\begin{array}{l}\text { Level III } \\
\text { Grade B }\end{array}$ \\
\hline 35 & $\begin{array}{l}\text { Russo-Ma } \\
\text { gno, P. } \\
(2001)\end{array}$ & $\begin{array}{l}\text { Retrospect } \\
\text { ive Chart } \\
\text { Review }\end{array}$ & $\begin{array}{l}-33 \text { older male } \\
\text { patients diagnosed } \\
\text { with OSA- } \\
\text { Setting-Veterans } \\
\text { Affairs Medical } \\
\text { Center }\end{array}$ & $\begin{array}{l}\text {-Patients who attended group sessions (discussed diagnosis of OSA } \\
\text { and use of CPAP, mailed reminders of education and support } \\
\text { sessions, symptomatic patients given proper interventions needed) } \\
\text { had increased compliance to CPAP }\end{array}$ & $\begin{array}{l}\text {-Small sample size } \\
\text {-Sample only included } \\
\text { older males }\end{array}$ & $\begin{array}{l}\text { Level III } \\
\text { Grade B }\end{array}$ \\
\hline 20 & $\begin{array}{l}\text { Bollig, S. } \\
\text { (2010) }\end{array}$ & $\begin{array}{l}\text { Literature } \\
\text { Review by } \\
\text { a provider }\end{array}$ & N/A & $\begin{array}{l}\text {-Improving CPAP compliance is a multi-layered approach. Intensive } \\
\text { behavioral interventions and intensive patient education can improve } \\
\text { CPAP compliance } \\
\text {-Change to Auto-CPAP or BiPAP has shown some improvement of } \\
\text { compliance in some subsets }\end{array}$ & $\begin{array}{l}\text {-Did not specify } \\
\text { articles that were } \\
\text { reviewed }\end{array}$ & $\begin{array}{l}\text { Level V } \\
\text { Grade B }\end{array}$ \\
\hline 32 & $\begin{array}{l}\text { Pruitt, B. } \\
\text { (2009) }\end{array}$ & $\begin{array}{l}\text { Literature } \\
\text { Review }\end{array}$ & N/A & $\begin{array}{l}\text {-Early and ongoing education, good experience with CPAP titration, } \\
\text { immediate individual follow up, monitoring compliance and efficacy, } \\
\text { long term support and trouble shooting, mask fit and choice of } \\
\text { interface, nasal steroids/antihistamines, humidification, and group } \\
\text { support have all been shown to increase CPAP compliance }\end{array}$ & $\begin{array}{l}\text {-Did not review high } \\
\text { level literature } \\
\text {-Overall summary of } \\
\text { interventions data not } \\
\text { presented }\end{array}$ & $\begin{array}{l}\text { Level V } \\
\text { Grade C }\end{array}$ \\
\hline 17 & $\begin{array}{l}\text { Anstead, } \\
\text { M. } \\
\text { (1998) }\end{array}$ & $\begin{array}{l}\text { Literature } \\
\text { Review }\end{array}$ & N/A & $\begin{array}{l}\text {-Patient education and close patient follow up have been shown to } \\
\text { increase CPAP compliance }\end{array}$ & $\begin{array}{l}\text {-Not specific in articles } \\
\text { that were included in } \\
\text { literature review }\end{array}$ & $\begin{array}{l}\text { Level V } \\
\text { Grade B }\end{array}$ \\
\hline 43 & $\begin{array}{l}\text { Wickwire } \\
\text {, E. } \\
\text { (2012) }\end{array}$ & $\begin{array}{l}\text { Literature } \\
\text { Review }\end{array}$ & N/A & $\begin{array}{l}\text {-Cognitive Behavioral Therapy has been shown to increase CPAP } \\
\text { compliance }\end{array}$ & $\begin{array}{l}\text {-Limit of studies } \\
\text { reviewed }\end{array}$ & $\begin{array}{l}\text { Level V } \\
\text { Grade B }\end{array}$ \\
\hline
\end{tabular}

As shown in Table 1, the summary of the evidence that was reviewed. 


\section{Summary of the evidence}

Out of the 28 articles that were evaluated, fifteen were randomized control trials and one was a systematic review of randomized control trials, which according to the Johns Hopkins pyramid of evidence, is the highest level of evidence ${ }^{[12]}$. Three articles evaluated were quasi-experimental which according the Johns Hopkins pyramid of evidence is level 2 evidence ${ }^{[12]}$. Of the articles evaluated, five were observational studies and the final four were literature reviews by providers, which categorizes them into evidence levels three and five respectively ${ }^{[12]}$. Each of the articles was graded individually for quality. The grading definitions used for all articles were from the Johns Hopkins Nursing EvidenceBased Practice Research Evidence Appraisal grading tool. Grade A articles had consistent results, sufficient sample size, adequate control, definitive conclusions, and consistent recommendations based on extensive literature review that includes thoughtful reference to scientific evidence ${ }^{[12]}$. Grade B articles had reasonably consistent results, sufficient sample size, some control, and fairly definitive conclusions, reasonable consistent recommendations on fairly comprehensive literature review that includes some reference to scientific evidence ${ }^{[12]}$. Grade $\mathrm{C}$ articles had little evidence with inconsistent results, insufficient sample size, and firm conclusions can't be drawn from the article ${ }^{[12]}$. Grade C articles were kept if they were valuable to help answer which interventions are most effective in improving CPAP compliance.

The Consort 2010 Checklist was used to grade the quality of each randomized control trial that was evaluated ${ }^{[13]}$. Out of the fifteen studies, seven articles were grade A, five were grade B and three were grade C. The Critical Appraisal Skills Programme (CASP) grading tool was used to evaluate the systematic review, which was a Grade $\mathrm{A}^{[14]}$. The TREND Statement Checklist, which stands for Transparent Reporting of Evaluations With Nonrandomized Designs, was used to evaluate the three quasi-experimental articles ${ }^{[15]}$. Two articles were grade $\mathrm{B}$ and one article was grade $\mathrm{C}$, these articles were lower in quality however were helpful in answering the proposed research question which is why they were retained. The observational studies were evaluated with the Strobe Statement Checklist ${ }^{[16]}$. These articles had overall high quality with 4 being grade A and one being Grade B. The four literature reviews were graded with The Johns Hopkins Nursing Evidence-Based Practice Non-Research Evidence Appraisal Tool, three were Grade B and one was grade C ${ }^{[12]}$. Although these articles are low in strength and quality, they were kept, as they were helpful in answering the research question. The overall strength of the evidence that was included was moderate to high as most of the articles evaluated were evidence levels one or two. The overall quality of the articles evaluated was moderate to high as most articles were either grade A or grade B evidence (see Table 1).

After the evidence was graded the articles of higher impact were the articles that were used to guide the synthesis of the evidence. The randomized control trials and the systematic review were the highest impact articles that were reviewed. The quantitative articles had a larger impact in the synthesis of data then the qualitative articles did. Despite this, most articles that were reviewed had similar conclusions of interventions that are successful in improving CPAP compliance.

\section{Synthesis of the evidence}

The data was synthesized by first evaluating the quantitative data. The quantitative data had two overwhelming themes of interventions that have been shown to improve CPAP compliance. A third theme was noted in the qualitative data however in the quantitative data this intervention was found to be controversial which is why it was not concluded in the recommendations. After evaluating both the quantitative and qualitative data the same themes emerged which is how the data was synthesized and recommendations were made.

After the evaluation of each article three overall themes were identified in improving CPAP compliance in adults diagnosed with OSA: increased patient education, intensive patient support and spousal collaboration. These have been shown to be helpful in improving CPAP compliance. The delivery of intensive patient education was looked at in many different ways. Patient education was provided in the form of group sessions, office visits, written literature, telephone 
encounters, videos as well as in presentations, all of which were shown to increase CPAP compliance. Although the method of delivering patient education differed among articles, the core information portrayed in each education method was similar. Within each method of communicating patient education information there were three parts: the diagnosis of OSA, the treatment of OSA and remedies to CPAP problems that patients may experience. Education was given on the definition and pathophysiology of OSA. The procedure to diagnose OSA was also highlighted. Side effects and consequences of untreated OSA were communicated to patients at length. The consequences of untreated OSA that were targeted included health risks as well as personal costs. The purpose and definition of CPAP was then highlighted. Finally, remedies to common CPAP problems were discussed at length. Overall, the evidence showed a strong link to intensive patient education and increased CPAP compliance.

The next theme of improving CPAP compliance was intensive patient support. Intensive patient support has been studied in many different ways however common themes emerged from the literature. Intensive patient support can vary in types including frequent office visits with the sleep provider, telemonitoring and phone conversations if patients have decreased compliance or frequent home visits. Each type of intensive support had similar activities that were performed during these sessions. CPAP compliance review was a part of the session, so the patient was able to monitor their progress and adherence with CPAP. The CPAP compliance report typically included hours per night mask was worn, amount of time there was mask leak and how effectively the patient's OSA is being treated by their CPAP. The clinician providing the patient support would then be able to give each patient feedback and motivation based on the compliance report generated. The presence of any side effects with CPAP was also addressed, and the clinician helped the patient remedy the issue. Equipment review was performed to ensure the patient had up to date supplies and replacements were ordered as needed. Finally, a mask fitting was performed as well to ensure that the patient was comfortable with the mask and that they were using and wearing the mask correctly. Overall intensive support was shown to moderately increase CPAP compliance.

Studies assessing the effect of spousal involvement on improving patient CPAP compliance have been controversial. Studies have looked at many different types of spousal involvement and its relationship to CPAP compliance. Spousal involvement in the form of support and collaboration has been noted to increase CPAP compliance in patients. Spousal support can range from a spouse encouraging the patient to use CPAP nightly to helping their spouse to clean the machine and equipment appropriately. Spousal collaboration has also been shown to increase CPAP compliance. Collaboration had the most effect when CPAP patients were having difficulties with their CPAP, and the spouse and patient work together to solve the problem. Studies have shown that spousal support and collaboration can help improve CPAP compliance however the data remains unclear in any other type of spousal involvement.

\section{Practice change}

Recent studies have revealed compliance with CPAP is not optimal, as noncompliance with CPAP significantly reduces the overall effectiveness of treatment of OSA; these patients are at risk for comorbid health conditions ${ }^{[7]}$. Improvement of CPAP compliance will positively impact patients, patient's families, communities and the health care system as a whole. Interventions to improve CPAP compliance are essential. From the three main interventions patient education had the most consistent, highest level, and highest quality of evidence, which demonstrated it, improves CPAP compliance (see Table 2). Of the articles evaluated seven of the level one articles, two of the level two, four of the level three, and three of the level five articles showed that increased education increases patient compliance with CPAP. Of the articles demonstrating that intensive patient education increased CPAP compliance, the overall strength and quality of those articles were moderate to high.

The evidence points to the necessity of the implementation of an intensive patient education program for all OSA patients. The education should start at the time the diagnosis of OSA is made, by thoroughly discussing the definition and pathophysiology of OSA. The complications of untreated OSA should be explained at length to the patient and the personalized treatment choices are to be discussed thoroughly with the patient. The definition and purpose of CPAP should 
be explained in great detail to each patient. The education should highlight the benefits of CPAP as well as ways to eliminate potential CPAP side effects that patients may experience. When the patient returns after their diagnostic and treatment sleep study to review their results, the same education points should be reviewed. It should be emphasized that the patient should call the clinic with any problems or concerns with CPAP usage. Based on the evidence, each visit should review the definition of OSA, complications of untreated OSA, purpose of CPAP, benefits of CPAP and ways to eliminate potential CPAP side effects. As research has shown that intensive education is the most effective way to address the problem of CPAP noncompliance, it is imperative that there be a practice change implemented to reflect this.

Table 2. Johns Hopkins Nursing Evidenced-based Practice Synthesis and Recommendations Tool EBP Practice Question: What is the most effective intervention to increase CPAP compliance among adult OSA patients? Date: $12 / 10 / 12$

\begin{tabular}{|c|c|c|c|}
\hline Category (Level Type) & $\begin{array}{l}\text { Total Number } \\
\text { of Sources/Level }\end{array}$ & $\begin{array}{l}\text { Overall Quality } \\
\text { Rating }\end{array}$ & $\begin{array}{l}\text { Synthesis of Findings } \\
\text { Evidence That Answers the EBP Question }\end{array}$ \\
\hline $\begin{array}{l}\quad \underline{\text { Level I }} \\
\text { Experimental study } \\
\text { Randomized Controlled Trial } \\
\text { (RCT) } \\
\text { Systematic review of RCTs } \\
\text { with or without meta analysis }\end{array}$ & 16 & $\begin{array}{l}\text { A-8, B-5, C-3 } \\
\text { Overall-A/B }\end{array}$ & $\begin{array}{l}\text {-Increased patient education has been shown to increase } \\
\text { CPAP compliance. Education includes information on } \\
\text { OSA diagnosis, symptoms and treatment as well as } \\
\text { information on CPAP and remedies for CPAP issues. } \\
\text {-Intensive patient support with frequent home visits } \\
\text { focusing on patient education has increased CPAP } \\
\text { compliance. }\end{array}$ \\
\hline $\begin{array}{l}\quad \text { Level II } \\
\text { Quasi-experimental studies } \\
\text { Systematic review of a } \\
\text { combination of RCTs and } \\
\text { quasi-experimental studies, or } \\
\text { quasi-experimental studies only, } \\
\text { with or without meta-analysis }\end{array}$ & 3 & $\begin{array}{l}\text { A-0, B-2, C-1 } \\
\text { Overall-B }\end{array}$ & $\begin{array}{l}\text {-Intensive patient education has been shown to increase } \\
\text { CPAP compliance. Education includes information on } \\
\text { OSA diagnosis, symptoms and treatment as well as } \\
\text { information on CPAP and remedies for CPAP issues. }\end{array}$ \\
\hline $\begin{array}{l}\qquad \underline{\text { Level III }} \\
\text { ·Non-experimental study } \\
\text { ·Systematic review of a } \\
\text { combination of RCTs, } \\
\text { quasi-experimental and } \\
\text { non-experimental studies, or } \\
\text { non-experimental studies only, } \\
\text { with or without meta-analysis } \\
\text {-Qualitative study or systematic }\end{array}$ & 5 & $\begin{array}{l}\text { A-4, B-1, C-0 } \\
\text { Overall-A }\end{array}$ & $\begin{array}{l}\text {-Intensive patient education including information on } \\
\text { diagnosis of OSA, consequences of untreated OSA, } \\
\text { treatment options for OSA, and a CPAP review } \\
\text { improved CPAP compliance } \\
\text {-Collaboration between the patient and his wife leads to } \\
\text { increased compliance with CPAP }\end{array}$ \\
\hline
\end{tabular}
review of qualitative studies, with or without meta-synthesis

\section{Level IV}

Opinion of respected authorities and/or reports of nationally recognized expert 0 N/A N/A committees/consensus panels based on scientific evidence

\section{Level V}

Evidence obtained from literature reviews, quality improvement, program evaluation, financial analysis, or 4 case reports A-0, B-3, C-1 -Improving CPAP compliance is a multi-layered approach. Intensive CBT and patient education have Opinion of nationally Overall-B been shown to increase CPAP compliance recognized expert(s) based on experiential evidence

\section{Recommendations Based on Evidence Synthesis and Selected Translation Pathway}

There is compelling evidence, which shows increased and intensive patient education can improve CPAP compliance in adults diagnosed with OSA. Patient education includes information on diagnosis of OSA, symptoms of OSA, treatment of OSA, complications that result from untreated OSA, information on CPAP, remedies for potential complications with CPAP usage.

As shown in Table 2, the synthesis of the evidence that was reviewed. 


\section{Conclusion}

In conclusion, CPAP is highly effective in treating OSA, however compliance is often suboptimal leaving these patients at risk for comorbid conditions, increased health care costs, negative effects on their families as well as the health care system as a whole. The evidence demonstrated that the most effective way to improve CPAP compliance is with patient education as well as patient support. To improve CPAP compliance interventions such as, increased education and patient support, are essential to implement in order to effectively treat patients diagnosed with OSA.

\section{References}

[1] Epstein, L., Kristo, D., Strollo, P., Friedman, N., Malhotra, A., Patil, S., et al. Clinical Guideline for evaluation, management and long-term care of obstructive sleep apnea in adults; J Clin Sleep Med. 2009; 5(3): 263-276. PMid:19960649

[2] Weaver, T., Sawyer, A. Adherence to continuous positive airway pressure treatment for obstructive sleep apnea: Implications for future interventions; Indian J Med Res. 2010; 131: 248-258.

[3] Siccoli, M., Pepperell, J. Kohler, M., Craig, S., Davies, R., Stradling, J. Effects of continuous positive airway pressure on quality of life in patients with moderate to severe obstructive sleep apnea: Data from a randomized control trial; Sleep. 2008; 31(11): 1551-1558. PMid:19014075

[4] Weaver, T., Grunstein, R. Adherence to continuous positive airway pressure therapy; Proc Am Thorac Soc. 2008 ; 5: 173-178. PMid:18250209 http://dx.doi.org/10.1513/pats.200708-119MG

[5] American Academy of Sleep Medicine; Positive airway pressure (PAP) devices-Physician frequently asked questions. Available from: http://www.aasmnet.org/Resources/PDF/PAPFAQs.pdf., 2008, accessed 11/13/12.

[6] Young, T., Peppard, P., Gottlieb, D. Epidemiology of obstructive sleep apnea; Am J Respir Crit Care Med. 2002; 165: 1217-1239. http://dx.doi.org/10.1164/rccm.2109080

[7] Weaver, T., et al. Relationship between hours of CPAP use and achieving normal levels of sleepiness and daily functioning; Sleep. 2007; 30(6): 711-719. PMid:17580592

[8] Simon-Tuval, T., Reuveni, H., Greenberg-Dotan, S., Oksenberg, A., Tal, A., Tarasiuk, A. Low socioeconomic status is a risk factor for CPAP acceptance among adult OSAS patients requiring treatment; Sleep. 2009; 32(4): 545-552.

[9] Wittmann, V., Rodenstein, D. Health care costs and sleep apnea syndrome; Sleep Med Rev. 2004; 4: 269-279. PMid:15233955 http://dx.doi.org/10.1016/j.smrv.2004.01.002

[10] Rodenstein, D. Sleep apnea: Traffic and occupational accidents-individual risks, socioeconomic and legal implications; Respiration. 2009; 78: 241-248. PMid:19494476 http://dx.doi.org/10.1159/000222811

[11] Boss, C. Searching for evidence: mission-critical tips; Nursing Management. 2009; 40(9): 12-14. PMid:19734750 http://dx.doi.org/10.1097/01.NUMA.0000360766.29235.8e

[12] Dearholt, S., Dang, D. Johns Hopkins Nursing Evidence-Based Practice Model and Guidelines; Indiana: Renee Wilmeth; 2012. PMid:22970528

[13] Schulz, K. F., Altman, D. G., Moher, D. CONSORT 2010 statement: updated guidelines for reporting parallel group randomized trials; Open Med. 2010; 4(1): e60. PMid:21686296

[14] Critical Appraisal Skills Programme (CASP); Critical Appraisal Skills Programme: Making sense of evidence about clinical effectiveness. Available from: http://www.casp-uk.net/, 10/31/12.

[15] Des Jarlais, D. C., Lyles, C., Crepaz, N., the Trend Group; Improving the reporting quality of nonrandomized evaluations of behavioral and public health interventions: The TREND statement; Am J Public Health. 2004; 94: 361-366. PMid:14998794 http://dx.doi.org/10.2105/AJPH.94.3.361

[16] Von Elm, E., Altman, D. G., Egger, M., Pocock, S. J., Gøtzsche, P. C., Vandenbroucke, J. P. The Strengthening the Reporting of Observational Studies in Epidemiology (STROBE) statement: guidelines for reporting observational studies; Prev Med. 2007; 45(4): 247-251. PMid:17950122 http://dx.doi.org/10.1016/j.ypmed.2007.08.012

[17] Anstead, M., Phillips, B., \& Buch, K. Tolerance and intolerance to continuous positive airway pressure. Current Opinion in Pulmonary Medicine. 1998; 4(6): 351-354. PMid:10813215 http://dx.doi.org/10.1097/00063198-199811000-00008

[18] Ballard, R. D., Gay, P. C., \& Strollo, P. J. Interventions to improve compliance in sleep apnea patients previously non-compliant with continuous positive airway pressure. Journal of Clinical Sleep Medicin: JCSM: Official Publication of the American Academy of Sleep Medicine. 2007; 3(7): 706-712.

[19] Baron, K., Gunn, H., Czajkowski, L., Smith, T., \& Jones, C. Spousal involvement in CPAP: Does pressure help? Journal of Clinical Sleep Medicine. 2012; 8(2): 147-153. 
[20] Bollig, S. M. Encouraging CPAP adherence: It is everyone's job. Respiratory Care. 2010; 55(9): 1230-1239. PMid:20800003

[21] Cartwright, R. Sleeping together: A pilot study of the effects of shared sleeping on adherence to CPAP treatment in obstructive sleep apnea. Journal of Clinical Sleep Medicine: JCSM: Official Publication of the American Academy of Sleep Medicine. 2008; 4(2): 123-127. PMid:18468310

[22] Chervin, R. D., Theut, S., Bassetti, C., \& Aldrich, M. S. Compliance with nasal CPAP can be improved by simple interventions. Sleep. 1997; 20(4): 284-289. PMid:9231954

[23] Damjanovic, D., Fluck, A., Bremer, H., Muller-Quernheim, J., Idzko, M., \& Sorichter, S. Compliance in sleep apnoea therapy: Influence of home care support and pressure mode. The European Respiratory Journal: Official Journal of the European Society for Clinical Respiratory Physiology. 2009; 33(4): 804-811. http://dx.doi.org/10.1183/09031936.00023408

[24] DeMolles, D. A., Sparrow, D., Gottlieb, D. J., \& Friedman, R. A pilot trial of a telecommunications system in sleep apnea management. Medical Care. 2004; 42(8): 764-769. PMid:15258478 http://dx.doi.org/10.1097/01.mlr.0000132353.99209.fe

[25] Fox, N., Hirsch-Allen, A., Goodfellow, E., Wenner, J., Fleetham, J., Ryan, C., et al. The impact of a telemedicine monitoring systen on positive airway pressure adherence in patients with obstructive sleep apnea: A randomized controlled trial. Sleep. 2012; 35(4): 477-481. PMid:22467985

[26] Fuchs, F. S., Pittarelli, A., Hahn, E. G., \& Ficker, J. H. Adherence to continuous positive airway pressure therapy for obstructive sleep apnea: Impact of patient education after a longer treatment period. Respiration; International Review of Thoracic Diseases. 2010; 80(1): 32-37. PMid:19786730 http://dx.doi.org/10.1159/000243161

[27] Golay, A., Girard, A., Grandin, S., Metrailler, J. C., Victorion, M., Lebas, P., et al. A new educational program for patients suffering from sleep apnea syndrome. Patient Education and Counseling. 2006; 60(2): 220-227. PMid:16253467 http://dx.doi.org/10.1016/j.pec.2005.01.007

[28] Hoy, C. J., Vennelle, M., Kingshott, R. N., Engleman, H. M., \& Douglas, N. J. Can intensive support improve continuous positive airway pressure use in patients with the sleep apnea/hypopnea syndrome? American Journal of Respiratory and Critical Care Medicine. 1999; 159(4 Pt 1): 1096-1100. PMid:10194151 http://dx.doi.org/10.1164/ajrccm.159.4.9808008

[29] Likar, L. L., Panciera, T. M., Erickson, A. D., \& Rounds, S. Group education sessions and compliance with nasal CPAP therapy. Chest. 1997; 111(5): 1273-1277. PMid:9149582 http://dx.doi.org/10.1378/chest.111.5.1273

[30] Massie, C. A., Hart, R. W., Peralez, K., \& Richards, G. N. Effects of humidification on nasal symptoms and compliance in sleep apnea patients using continuous positive airway pressure. Chest. 1999; 116(2): 403-408. PMid:10453869 http://dx.doi.org/10.1378/chest.116.2.403

[31] Pamidi, S., Knutson, K. L., Ghods, F., \& Mokhlesi, B. The impact of sleep consultation prior to a diagnostic polysomnogram on continuous positive airway pressure adherence. Chest. 2012; 141(1): 51-57. PMid:21700685 http://dx.doi.org/10.1378/chest.11-0709

[32] Pruitt, B. Top 10 practices to increase CPAP compliance. RT: The Journal for Respiratory Care Practitioners. 2009 ; $22-25$.

[33] Richards, D., Bartlett, D. J., Wong, K., Malouff, J., \& Grunstein, R. R. Increased adherence to CPAP with a group cognitive behavioral treatment intervention: A randomized trial. Sleep. 2007; 30(5): 635-640. PMid:17552379

[34] Roecklein, K., Schumacher, J., Gabriele, J., Fagan, J., Baran, A., \& Richert, A. Personalized feedback to improve CPAP adherence in obstructive sleep apnea. Behavioral Sleep Medicine. 2010; 8: 105-112. PMid:20352546 http://dx.doi.org/10.1080/15402001003622859

[35] Russo-Magno, P., O'Brien, A., Panciera, T., \& Rounds, S. Compliance with CPAP therapy in older men with obstructive sleep apnea. Journal of the American Geriatrics Society. 2001; 49(9): 1205-1211. PMid:11559380 http://dx.doi.org/10.1046/j.1532-5415.2001.49238.x

[36] Silva, R. S., Truksinas, V., de Mello-Fujita, L., Truksinas, E., Zanin, L. K., Pinto, M. C., et al. An orientation session improves objective sleep quality and mask acceptance during positive airway pressure titration. Sleep \& Breathing = Schlaf \& Atmung. 2008; 12(1): 85-89. PMid:17924157 http://dx.doi.org/10.1007/s11325-007-0138-6

[37] Smith, I., Lasserson, T., \& Haniffa, M. Interventions to improve use of continuous positive airway pressure for obstructive sleep apnoea (review). The Cochrane Collaboration. 2009; (3).

[38] Smith, C. E., Dauz, E., Clements, F., Werkowitch, M., \& Whitman, R. Patient education combined in a music and habit-forming intervention for adherence to continuous positive airway (CPAP) prescribed for sleep apnea. Patient Education and Counseling. 2009; 74(2): 184-190. PMid:18829212 http://dx.doi.org/10.1016/j.pec.2008.08.008

[39] Smith, C. E., Dauz, E. R., Clements, F., Puno, F. N., Cook, D., Doolittle, G.,et al. Telehealth services to improve nonadherence: A placebo-controlled study. Telemedicine Journal and e-Health: The Official Journal of the American Telemedicine Association. 2006; 12(3): 289-296. PMid:16796496 http://dx.doi.org/10.1089/tmj.2006.12.289 
[40] Sparrow, D., Aloia, M., Demolles, D. A., \& Gottlieb, D. J. A telemedicine intervention to improve adherence to continuous positive airway pressure: A randomised controlled trial. Thorax. 2000; 65(12): 1061-1066. PMid:20880872 http://dx.doi.org/10.1136/thx.2009.133215

[41] Stepnowsky, C. J., Palau, J. J., Marler, M. R., \& Gifford, A. L. Pilot randomized trial of the effect of wireless telemonitoring on compliance and treatment efficacy in obstructive sleep apnea. Journal of Medical Internet Research. 2007; 9(2): e14. PMid:17513285

[42] Trupp, R. J., Corwin, E. J., Ahijevych, K. L., \& Nygren, T. The impact of educational message framing on adherence to continuous positive airway pressure therapy. Behavioral Sleep Medicine. 2011; 9(1): 38-52. PMid:21218293 http://dx.doi.org/10.1080/15402002.2011.533993

[43] Wickwire, E. A promising intervention; improving positive airway pressure adherence with cognitive-behavioral treatment. RT: The Journal for Respiratory Care Practitioners. 2012; 14-17.

[44] Wiese, H., Boethel, C., Phillips, B., Wilson, J., Peters, J., Viggiano, T. CPAP compliance: Video education may help! Sleep Medicine. 2005; 6(2): 171-174. PMid:15716221 http://dx.doi.org/10.1016/j.sleep.2004.08.006 